\title{
Numerical simulation of the wind device for manned agricultural aircraft
}

\author{
Meng Sun ${ }^{1,3}$, Liqun $\mathrm{Lu}^{1,3^{*}}$, Yubin Lan ${ }^{2,3}$, Jing Zhao ${ }^{2,3}$, Gang Fang ${ }^{1,3}$ \\ (1. School of Traffic and Vehicle Engineering, Shandong University of Technology, Zibo 255000, Shandong, China; \\ 2. School of Agricultural Engineering and Food Science, Shandong University of Technology, Zibo 255000, Shandong, China; \\ 3. National Sub-center for International Collaboration Research on Precision Agricultural Aviation Pesticide Spraying Technology, \\ Shandong University of Technology Sub-center of National Center for International Collaboration Research on Precision Agricultural \\ Aviation Pesticide Spraying Technology, Zibo 255000, China)
}

\begin{abstract}
In order to reduce the energy consumption of an agricultural aircraft, a kind of pneumatic and energy storage system was designed to drive the aircraft operation, including pneumatic device, compressed air device and energy storage device. As the prime mover of the whole system, the pneumatic device rotates under the action of high-speed wind energy generated during aircraft flight to drive the compressor to work and convert mechanical energy into compressed air energy, and the energy storage device is used to store residual energy. Different installation positions of the pneumatic device have different degrees of influence on the flight flow field. In order to determine the best installation position of the wind device on the manned agricultural aircraft, a certain type of agricultural aircraft was used as an example, and the appearance model of the aircraft was designed. The pneumatic device was installed in front of the wing, behind the wing and under the wing for analysis, so as to obtain the best installation position. At the best position, the variation law of output torque of pneumatic impeller under different wind speed conditions was investigated. The results show that the installation of the wind device in front of the wing has the least impact on the aircraft and the pneumatic impeller, and the output torque of the pneumatic impeller is directly related to the aircraft wind speed, angle of attack and flight speed. It decreases with the increase of the angle of attack at 0,6 and 12 angles of attack, and increases with the increase of the wind speed at $40 \mathrm{~m} / \mathrm{s}, 50 \mathrm{~m} / \mathrm{s}$ and $60 \mathrm{~m} / \mathrm{s}$.
\end{abstract}

Keywords: pneumatic device, flight angle of attack, lift coefficient, the drag coefficient

DOI: $10.33440 /$ j.ijpaa.20210402.175

Citation: Sun M, Lu L Q, Lan Y B, Zhao J, Fang G. Numerical simulation of the wind device for manned agricultural aircraft. Int J Precis Agric Aviat, 2021; 4(2): 22-29.

\section{Introduction}

At present, the power of manned agricultural aircraft and its operating system is provided by its own engine consumption of oil or natural gas and other non-renewable energy, when tens to hundreds of tons of aircraft fly in the air, the fuel consumption per hour will reach several tons or even ten tons, and the energy consumption is huge. With the increasingly tight supply of aviation gasoline, new requirements are put forward for the energy consumption of aircraft, which highlights the importance of reducing the energy consumption of agricultural aircraft.

During the flight, the speed of the aircraft can reach 250$300 \mathrm{~km} / \mathrm{h}^{[1]}$, which generates considerable wind energy. Making full use of this part of wind energy is conducive to improving the energy utilization efficiency of the aircraft. For the research on the utilization of wind energy on the aircraft, domestic and foreign scholars have made beneficial attempts and explorations: Italy of Augusta westland company invented a technique called "Project

\section{Received date: 2021-09-10 Accepted date:2021-10-22}

Biographies: Meng Sun, Postgraduate student, research interests: vehicle multi power and electro-hydraulic control technology. Email: sunmeng0121@ gmail.com; Yubin Lan, phD, professor, Director, research interests: precision agricultural aviation application, Email: ylan@scau.edu.cn; Jing Zhao, Associate professor research interests: remote sensing, Email: zbceozj@ 163.com; Gang Fang, Postgraduate student, research interests: vehicle multi power and electro-hydraulic control technology, Email: 977514164@qq.com.

* Corresponding author: Liqun Lu, Associate professor, research interests: vehicle multi power and electro-hydraulic control technology, Mailing Address: Shandong University of Technology West Campus. Email: luliqun@sdut.edu.cn.
Zero" new electric tilting rotor technology demonstration aircraft, this invention abandons the hydraulic system on ordinary aircraft and uses an electric motor powered by rechargeable batteries to provide power for the aircraft, the electric energy of the battery is generated by the high-speed rotation of the aircraft's rotor in the wind, making the greatest use of the wind energy generated by flight $^{[2,3]}$. In 2017, Xsens in the Netherlands developed a mooring unmanned aircraft (UAV) that uses wind energy as the original power generation at higher altitudes ${ }^{[4,5]}$. The UAV connects the fuselage and the generator on the ground with a mooring rope. When the aircraft flies up autonomously under the action of wind, the mooring rope drives the generator to generate electricity. When the flight exceeds the length of the tether, the aircraft will automatically descend and make the tether rewind, and then rise again after rewind. In this way, the round-trip can continuously generate electricity with the help of wind energy. Tang Wenchuan $^{[6]}$ from AVIC Tongfei Research Institute limited company, analyzed the pneumatic pump from the technical origin, technical principle, technical advantages and characteristics, applications, limitations and other dimensions, and concluded that the pneumatic pump technology can be used in a wide range of aircraft, which made a beneficial exploration for the application of pneumatic technology. $\mathrm{Li} \mathrm{Lin}^{[7]}$ of Beijing University of Aeronautics and Astronautics analyzed and calculated the basic structural parameters of the centrifugal fan of the helicopter cooling system according to the flight conditions of the helicopter and the working environment of the centrifugal fan. According to the "controllable vortex" design theory, she aerodynamic designed the rotary surface profile of the centrifugal 
fan, and put forward the universal design program of the centrifugal fan, the fan efficiency is effectively improved while simplifying the design process.

Power system using wind energy to drive operation on aircraft, the key component is the pneumatic device. The pneumatic device can rotate at high speed under the action of the wind energy generated by the flight, drive the compressor to work, and convert mechanical energy into compressed air energy. However, there is also a pneumatic influence between the air device and the aircraft, which reduces the utilization efficiency of the air device. In this paper, the influence law of the installation position of the air device on the agricultural aircraft and itself and the research of the air device under different wind speeds are analyzed to reduce the aerodynamic interference between the agricultural aircraft and the air device.

\section{Force analysis of agricultural aircraft}

\subsection{Modeling of agricultural aircraft}

In order to study the interaction law of aerodynamic performance between the aircraft and the wind device, a certain type of agricultural aircraft was selected as the research object. NACA4415 was used for the wing, the installation angle of the wing was $-2.87^{\circ}$, and NACA0012 was used for the vertical tail and flat tail airfoil. The specific design parameters are shown in Table 1.

Table 1 Design parameters of manned agricultural aircraft

\begin{tabular}{lc}
\hline \multicolumn{1}{c}{ Design project } & Project Parameters \\
\hline The wing chord length & $1.94 \mathrm{~m}$ \\
Long wings show & $14.97 \mathrm{~m}$ \\
Flat tail span & $4.93 \mathrm{~m}$ \\
Flat tail chord length & $1.11 \mathrm{~m}$ \\
Vertical tail span & $1.54 \mathrm{~m}$ \\
Vertical tail root chord length & $1.73 \mathrm{~m}$ \\
Vertical tail tip chord length & $0.82 \mathrm{~m}$ \\
Reference area & $31.7 \mathrm{~m}^{2}$ \\
\hline
\end{tabular}

For the convenience of research, the influence of aircraft propeller slipflow is ignored, and the propeller and its accessories are omitted. According to the structure and design parameters of the aircraft, reverse engineering is used to obtain the appearance of the aircraft in SolidWorks, as shown in Figure 1.

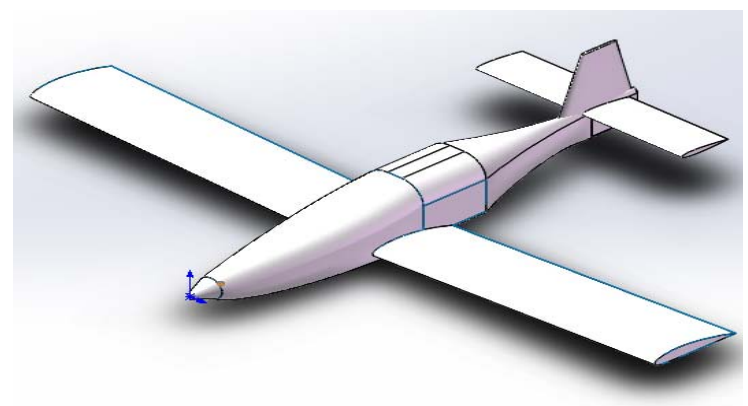

Figure 1 Three-dimensional model of agricultural aircraft

\subsection{Aircraft stress analysis}

The aircraft is regarded as a rigid body, and its forces during flight are shown in Figure 2, which mainly include the gravity of the aircraft itself $G$, the thrust of the engine $T$, the lift force perpendicular to the velocity direction $L$, the drag force parallel to the velocity direction $D$, the side force perpendicular to the flight plane of the aircraft $C$, and the forces of the landing gear and the ground during take-off and landing. Lift force $L$, drag force $D$ and side force $C$ are collectively referred to as aerodynamics of the aircraft ${ }^{[8]}$.

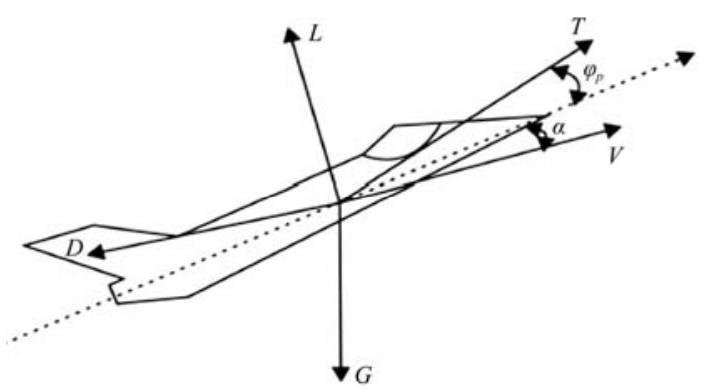

Figure 2 Aircraft flight stress map

The core of aerodynamic analysis of aircraft is to solve the aerodynamic coefficient of aircraft. The relationship between aerodynamic coefficient and aerodynamic force (lift force $L$, drag force $D$, side force $C$ ) can be expressed in Equations (1), (2) and (3) respectively.

$$
\begin{aligned}
L & =\frac{1}{2} C_{L} S \rho v^{2} \\
D & =\frac{1}{2} C_{D} S \rho v^{2} \\
C & =\frac{1}{2} C_{C} S \rho v^{2}
\end{aligned}
$$

where, $C_{L}$ is the lift coefficient; $C_{D}$ is the drag coefficient; $C_{C}$ is the lateral force coefficient, and $S$ is the aircraft reference area.

\subsection{Structural design of pneumatic device}

The role of the pneumatic device in the wind-energy storage system is to make full use of the high-speed wind energy generated during the flight of agricultural aircraft, and drive the compressor to compress the air for energy storage. As the prime mover of the whole system, the form and parameters of the impeller blades will have an important impact on the system. Assuming that the wind speed is $40 \mathrm{~m} / \mathrm{s}$ and the design power is $3.5 \mathrm{~kW}$, the aerodynamic shape of the blade is designed to determine the basic aerodynamic parameters of the blade, including blade radius, blade number, selection of airfoil, chord length, installation angle, tip speed ratio and so on.

\subsubsection{Blade radius}

The blade radius is generally determined by Equation (4):

$$
R=\sqrt{\frac{2 P}{\pi \eta \rho C_{P} V_{0}^{3}}}
$$

where, $R$ is the blade radius; $P$ is the output power of wind turbine; $\eta$ is the efficiency of the entire wind energy utilization system, and the value is $0.85 ; \rho$ is air density, the value is $1.225 \mathrm{~kg} / \mathrm{m}^{3} ; C_{p}$ is the utilization coefficient of wind energy, the initial value is 0.41 according to the design experience, and the actual size is obtained according to the actual output power of the blade; $V_{0}$ is the working wind speed.

The blade radius was calculated to be $0.29 \mathrm{~m}$. Considering the accuracy of installation and machining, the radius was set to $0.3 \mathrm{~m}$.

2.3.2 Number of blades and tip speed ratio

The sharp speed ratio is generally determined by wind turbine type, blade number and other parameters ${ }^{[9,10]}$. The number of blades with high wind speed corresponds to the range of sharp speed ratio of 6-8, which can better capture wind energy. So the sharp speed ratio designed in this paper is 7 . According to Table 2 , the number of blades is 3 . 


\subsubsection{Determination of airfoil}

Generally, the ratio of lift coefficient $C_{L}$ and drag coefficient $C_{D}$ is used to evaluate the wind energy utilization efficiency of airfoil. The greater the ratio, the better the wind energy utilization efficiency will be. The airfoil type selected in this paper is NACA4412. The lift-drag ratio curve and pitching moment curve of this airfoil at an angle of attack of $-8^{\circ}$ to $-13^{\circ}$ are obtained by professional airfoil analysis software Profili, as shown in Figure 3. It can be seen that the lift-drag ratio of this airfoil reaches the maximum at an angle of attack of $6^{\circ}$, which is about 100.4.

The final parameters of the vane are shown in Table 3.
Table 2 Corresponding table of tip speed ratio and blade number

\begin{tabular}{ccc}
\hline Tip speed ratio & Number of blades & Type of wind turbine \\
\hline 1 & $8-24$ & Low speed wind turbine \\
2 & $6-12$ & \\
\hline 3 & $3-8$ & Medium speed wind turbine \\
4 & $3-5$ & \\
\hline $5-8$ & $2-4$ & High speed wind turbine \\
\hline $8-15$ & $1-2$ & \\
\hline
\end{tabular}
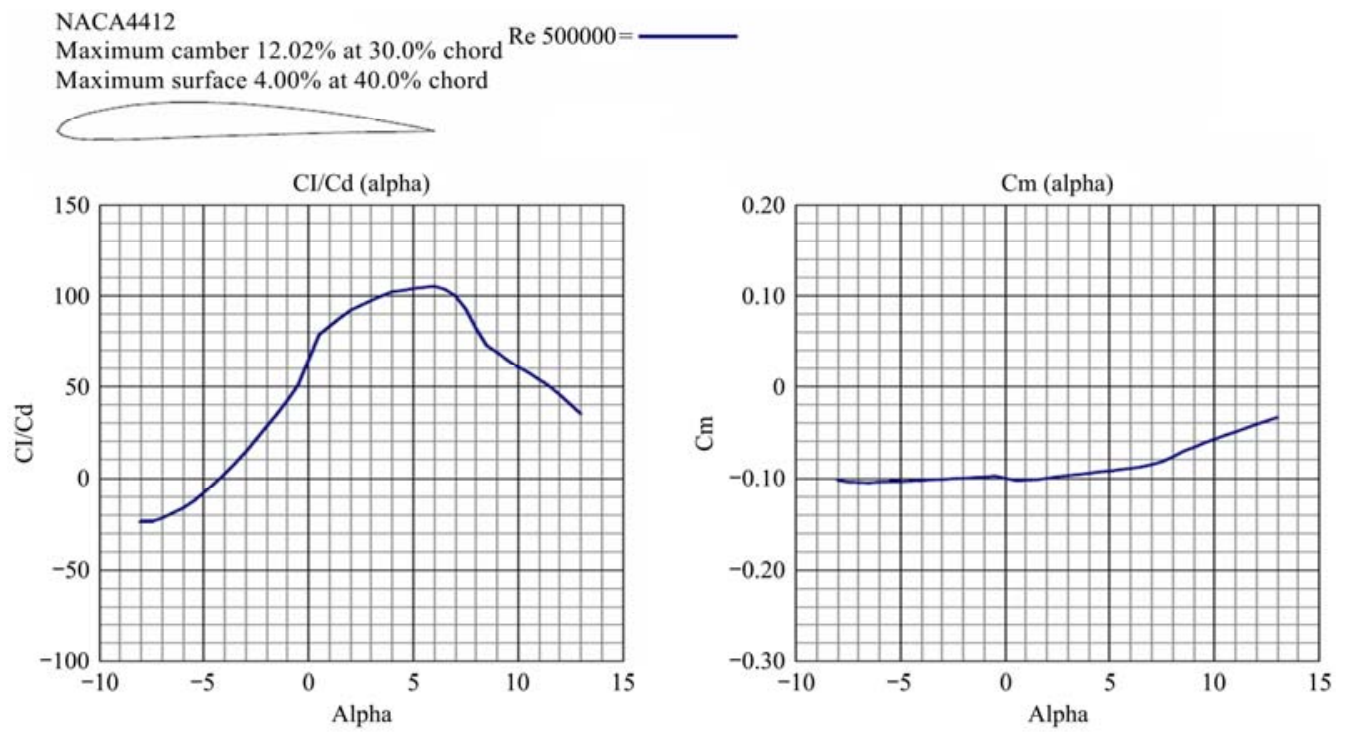

Figure 3 Lift-drag ratio curve (left) and pitch moment curve (right) of NACA4412 airfoil

Table 3 Vane parameter table of pneumatic device

\begin{tabular}{lc}
\hline The name of the & Parameter \\
\hline Leaf blade radius & $0.3 \mathrm{~m}$ \\
Leaf number & 3 \\
Airfoil & NACA4412 \\
The tip speed ratio & 7 \\
\hline
\end{tabular}

According to the basic parameters of the blade, 3D software was used to carry out mechanical design for a single blade, as shown in Figure 4. After forming a single blade model, assemble the three blades to obtain the assembly model of the entire impeller of the pneumatic device (hereinafter referred to as the impeller), as shown in Figure 5.

In order to avoid the collision between the lower fuselage of the aircraft and the impeller blades, and facilitate the release and retraction of the pneumatic device, the distance between the blade rotating wing tip and the lower fuselage is designed to be $300 \mathrm{~mm}$. The assembly drawing is shown in Figure 6.

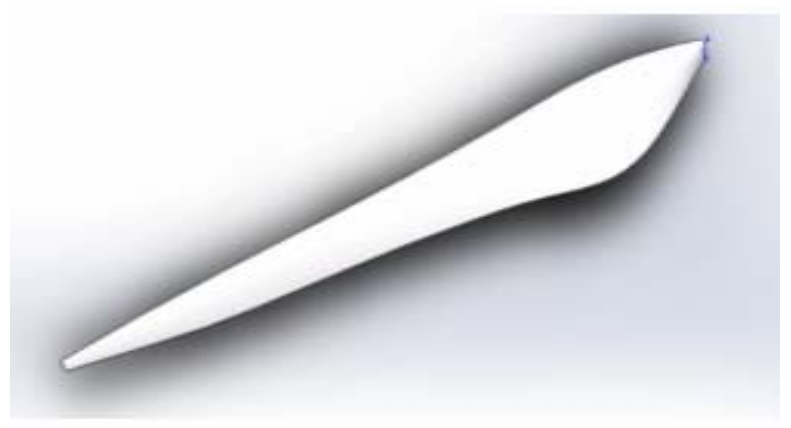

Figure 4 (single) Three-dimensional blade model

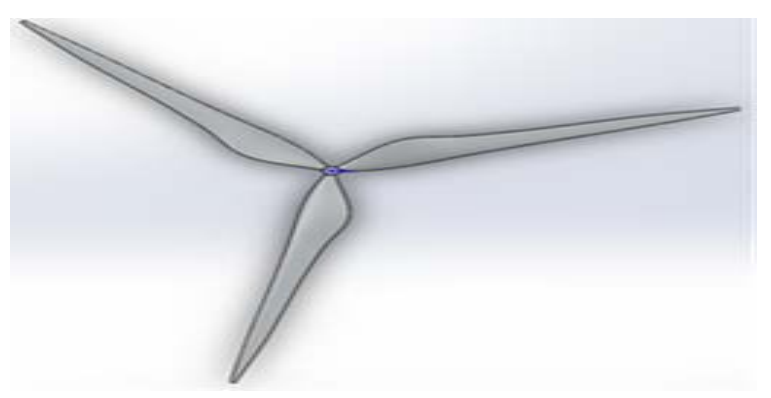

Figure 5 Pneumatic impeller assembly model

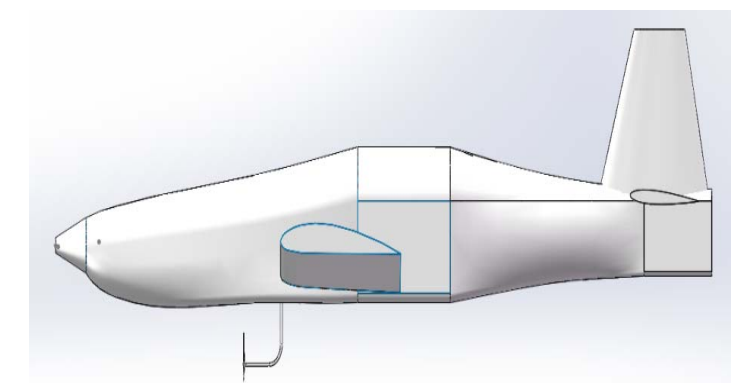

Figure 6 Assembly drawings of aircraft and pneumatic impeller

\section{Establishment of finite element model}

\subsection{Meshing and boundary conditions}

According to the structural characteristics of the aircraft and the impeller, the triangular mesh of the unstructured mesh is used and locally refined. When dividing, the whole computing domain is divided into two parts: one part is the rotation domain, which is the rotating region of the impeller, and the mesh number is 470,000 ; the other part is the static region, which is excluding the pneumatic 
impeller all the area, the grid number is 2.8 million. The static domain and the rotating domain transfer the data between the grids through the interface, and the distribution after mesh division is shown in Figure 7.

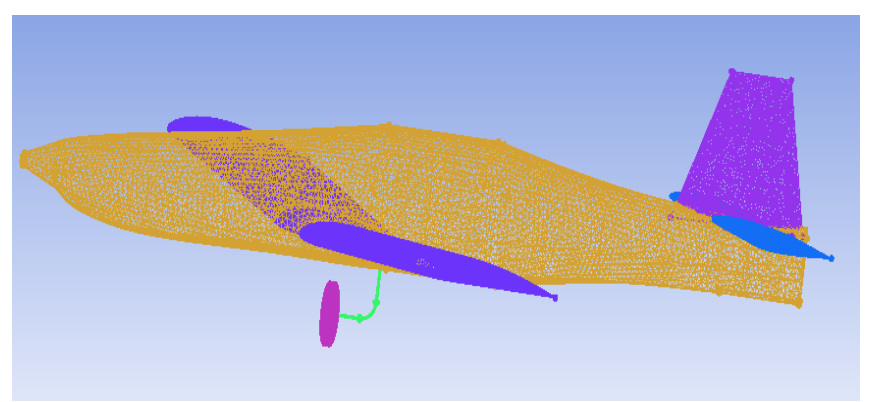

Figure 7 Mesh distribution of aircraft and pneumatic impellers

To ensure accuracy, boundary conditions need to be set with velocity-inlet, outflow, wall (wind impeller is rotating wall, aircraft fuselage is stationary wall), and interface. The distance between the inlet surface and the aircraft is one length of fuselage, and the distance between the outlet surface and the aircraft is five lengths of fuselage. The speed and direction of wind energy are set on the velocity inlet surface, the rotating speed and direction of the impeller are set on the wall of the impeller, and the flow medium is set according to the ideal air.

\subsection{Calculation method}

Assuming that the medium flowing through the aircraft and the pneumatic impeller is compressible ideal air and the flow process is adiabatic, the continuity equation (mass conservation law), momentum conservation equation and energy conservation equation are satisfied in the working process ${ }^{[11-13]}$.

(1) Equation of continuity

$$
\frac{\partial \rho}{\partial t}+\frac{\partial(\rho u)}{\partial x}+\frac{\partial(\rho v)}{\partial y}+\frac{\partial(\rho w)}{\partial z}=0
$$

where, $\rho$ is the fluid density; $t$ is time, and $u, v, w$ is the velocity component in $x, y$, and $z$.

(2) Momentum conservation equation

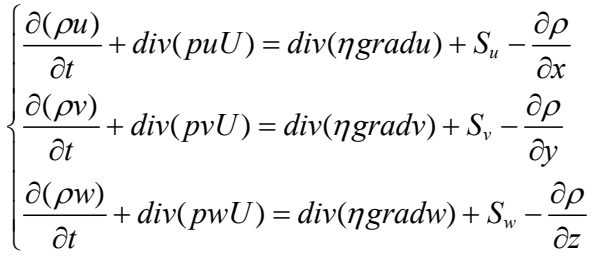

where, $S_{u}, S_{v}, S_{w}$ is the generalized source term of the momentum equation and $d i v$ is the divergence.

The momentum conservation law equations in fluid mechanics are called N-S equations (Navier-Stokes equations), which reveal the basic laws of viscous flow and are an important theoretical basis of fluid mechanics ${ }^{[14,15]}$.

(3) Energy conservation equation

$$
\frac{\partial(\rho T)}{\partial t}+\operatorname{div}(\rho u T)=\operatorname{div}\left(\frac{k}{c_{p}} \operatorname{grad} T\right)+S_{T}
$$

where, $T$ is the temperature; $k$ is the heat transfer coefficient; $C_{p}$ is the specific heat capacity, and $S_{T}$ is the viscous dissipation term.

Due to its high prediction of separated flows, the SST $k-\omega$ model has been widely used in the field of aerodynamic performance research of pneumatic components ${ }^{[10]}$. Therefore, the SST $k$ - $\omega$ model was selected as the turbulence model in this paper. The SST $k$ - $\omega$ model uses the $k$ - $\omega$ model for the calculation of the near wall area, and the $k-\omega$ model is used for the fully developed flow field area. The function expression of SST $k-\omega$ model is as follows:

$$
\begin{gathered}
\frac{\partial}{\partial}(\rho k)+\frac{\partial}{\partial}\left(\rho k u_{i}\right)=\frac{\partial}{\partial x_{j}}\left[\Gamma_{k} \frac{\partial k}{\partial x_{j}}\right]+\overline{G_{k}}-Y_{k}+S_{k} \\
\frac{\partial}{\partial}(\rho \omega)+\frac{\partial}{\partial}\left(\rho \omega u_{i}\right)=\frac{\partial}{\partial x_{j}}\left[\Gamma_{\omega} \frac{\partial \omega}{\partial x_{j}}\right]+\overline{G_{\omega}}-Y_{\omega}+S_{\omega}
\end{gathered}
$$

where, $k$ is the turbulent kinetic energy; $\omega$ is the turbulent energy dissipation rate; $\overline{G_{k}}$ is the production term resulting in $k$ due to the average velocity gradient; $\overline{G_{\omega}}$ is the production term of $\omega ; \quad \Gamma_{k}$ and $\Gamma_{\omega}$ are the diffusivity of $k$ and $\omega$, respectively; $Y_{k}$ and $Y_{\omega}$ are diffusion terms of $k$ and $\omega$, respectively; $D_{\omega}$ is the cross diffusion term; $S_{k}$ and $S_{\omega}$ are custom source items, respectively.

\section{Calculation results and analysis}

\subsection{Analysis of aircraft aerodynamic characteristics without pneumatic impeller}

In order to illustrate the influence of the pneumatic impeller on the aerodynamic performance of the aircraft, it is necessary to obtain the aerodynamic performance of the aircraft without the pneumatic impeller. Firstly, the plane mesh and volume mesh were generated for the aircraft model, and the unstructured mesh was divided, as shown in Figure 8 . In order to ensure the accuracy of calculation, cylindrical body is used in the outflow field. The inlet surface is one fuselage length from the aircraft, and the outlet surface is five fuselage length from the aircraft.

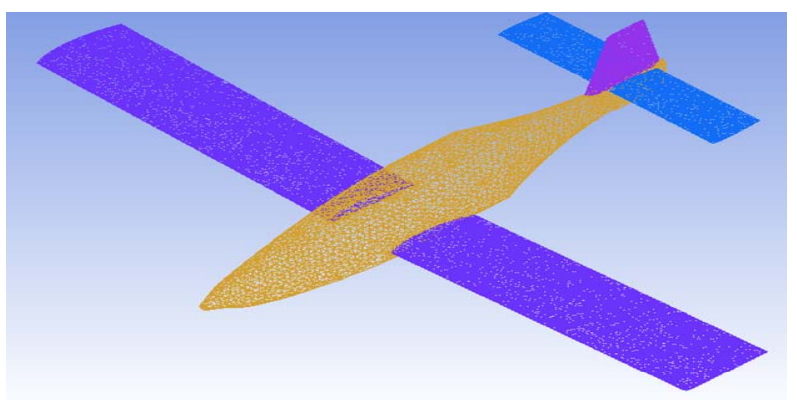

Figure 8 Grid distribution of agricultural aircraft models

Calculation state: the atmospheric state is the standard atmospheric state near the ground, the calculated angle of attack is $-2^{\circ}$ to $14^{\circ}$, and the incoming wind speed is $50 \mathrm{~m} / \mathrm{s}$. Table 4 shows the lift coefficient and drag coefficient of the aircraft without impeller.

Table 4 Lifting drag coefficient of the aircraft without impeller

The plane Angle of attack $\alpha /\left(^{\circ}\right)$ Lift coefficient $C_{L}$ Resistance coefficient $C_{D}$

$\begin{array}{ccc}-2 & 0.432 & 0.034 \\ 0 & 0.5711 & 0.046 \\ 2 & 0.634 & 0.062 \\ 4 & 0.704 & 0.082 \\ 6 & 0.810 & 0.101 \\ 8 & 0.881 & 0.126 \\ 10 & 0.975 & 0.154 \\ 12 & 1.036 & 0.183 \\ 14 & 1.036 & 0.213\end{array}$

4.2 Analysis of the interference of installation position of impeller of air device to agricultural aircraft

Calculation state: the atmospheric state is the standard atmospheric state near the ground, the angle of attack of the aircraft is calculated as $-2^{\circ}$ to $14^{\circ}$, the incoming wind speed is $50 \mathrm{~m} / \mathrm{s}$, and 
the installation positions of the pneumatic impeller are respectively in front of the wing, under the wing and behind the wing and a total of 27 calculation examples are carried out. The lift coefficient and drag coefficient of agricultural aircraft after calculation are shown in Table 5.

Table 5 Aircraft lift coefficient and drag coefficient in different installation positions of pneumatic impeller

\begin{tabular}{|c|c|c|c|c|c|c|}
\hline \multirow{2}{*}{$\begin{array}{c}\text { The plane angle } \\
\text { of attack } \\
\alpha /\left(^{\circ}\right)\end{array}$} & \multicolumn{2}{|c|}{ The front wing } & \multicolumn{2}{|c|}{ Below the wing } & \multicolumn{2}{|c|}{ The rear wing } \\
\hline & $C_{L}$ & $C_{D}$ & $C_{L}$ & $C_{D}$ & $C_{L}$ & $C_{D}$ \\
\hline-2 & 0.033 & 0.384 & 0.033 & 0.307 & 0.033 & 0.374 \\
\hline 0 & 0.042 & 0.437 & 0.043 & 0.414 & 0.042 & 0.468 \\
\hline 2 & 0.057 & 0.616 & 0.061 & 0.553 & 0.057 & 0.574 \\
\hline 4 & 0.069 & 0.735 & 0.075 & 0.585 & 0.073 & 0.656 \\
\hline 6 & 0.098 & 0.837 & 0.096 & 0.658 & 0.099 & 0.753 \\
\hline 8 & 0.121 & 0.922 & 0.121 & 0.735 & 0.119 & 0.87 \\
\hline 10 & 0.150 & 1.001 & 0.150 & 0.815 & 0.152 & 1.013 \\
\hline 12 & 0.184 & 1.106 & 0.186 & 0.921 & 0.174 & 1.029 \\
\hline 14 & 0.206 & 1.111 & 0.215 & 0.951 & 0.205 & 1.101 \\
\hline
\end{tabular}

The lift coefficient and drag coefficient of agricultural aircraft under the condition of no air impeller and under the condition of different installation positions are compared, as shown in the figure below. According to Figure 9, the pneumatic impeller has an influence on the lift coefficient at different positions of the agricultural aircraft, but it conforms to the aerodynamic law of the agricultural aircraft at different angles of attack. When the impeller is located under the wing, the influence reduces the lift coefficient of the aircraft to the maximum extent, indicating that the lift of agricultural aircraft decreases at this position, which should be avoided as much as possible during flight, so the pneumatic impeller should not be installed under the wing.

When the impeller is located in front of the wing and behind the wing, the influence on the lift coefficient of the aircraft is within a reasonable range. In the case of small angle of attack $\left(-2^{\circ}, 2^{\circ}\right)$ both reduce lift coefficient of the plane, but along with the increase of angle of attack, the impeller is located in the front wing would be advantageous to the aerodynamic characteristics of aircraft, aircraft lift coefficient were higher than the state without air lift coefficient of the impeller, which improved the lift, and pneumatic impeller is located in the rear wing is the opposite.

In these three cases, the installation position of the pneumatic impeller has no great influence on the lift coefficient of the aircraft, but reduces the drag coefficient of the aircraft in all cases, indicating that the existence of the pneumatic impeller can reduce

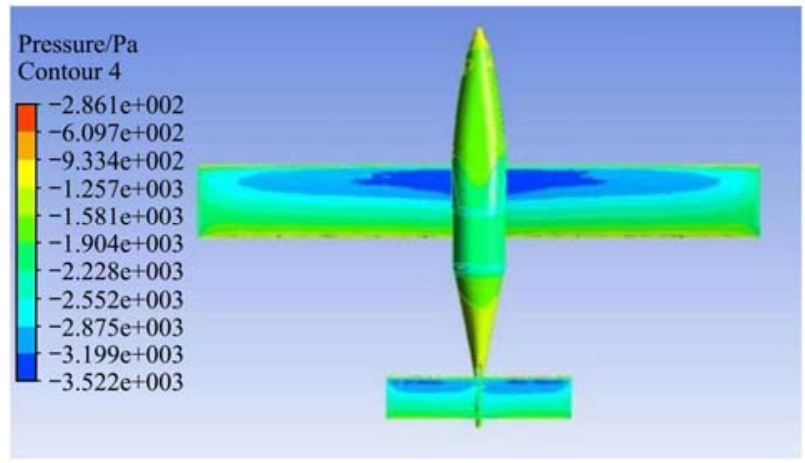

a. No impeller the drag in the flight process, which is beneficial to the aerodynamic characteristics of the aircraft.

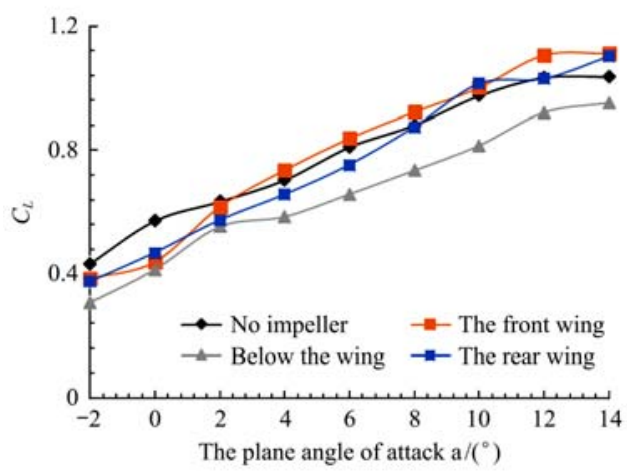

Figure 9 Lift coefficient of agricultural aircraft when the impeller of air device is not installed or installed in different positions

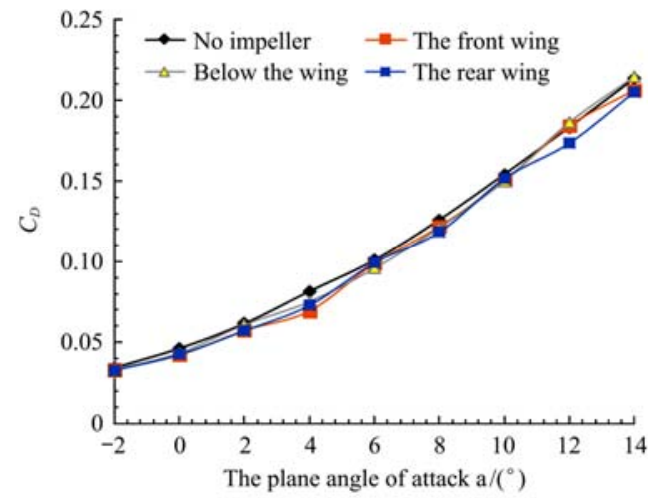

Figure 10 Drag coefficient of agricultural aircraft with no installation and different installation positions of impeller of air device

The experiment only analyzed the aircraft pressure distribution of the pneumatic impeller at different positions when the aircraft angle of attack was $0^{\circ}, 6^{\circ}$ and $12^{\circ}$ respectively, as shown in Figure 11, 12 and 13. It can be seen from the aircraft pressure distribution cloud diagram that the existence of the pneumatic impeller has different degrees of influence on the aircraft, especially the pressure of the wing. When the angle of attack is $0^{\circ}$, the presence of the pneumatic impeller greatly reduces the area of the minimum pressure surface of the aircraft wing, which is also the reason why the lift coefficient of the aircraft without the air impeller is greater than that with the air impeller when the angle of attack is $0^{\circ}$ as shown in Figure 9. With the increase of the angle of attack, the area of the minimum pressure surface of the wings of the three types of pneumatic impeller is basically unchanged.

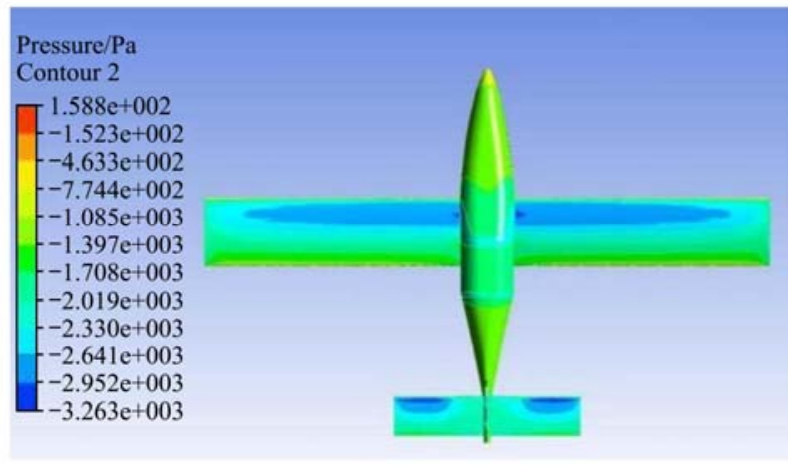

b. The front wing 


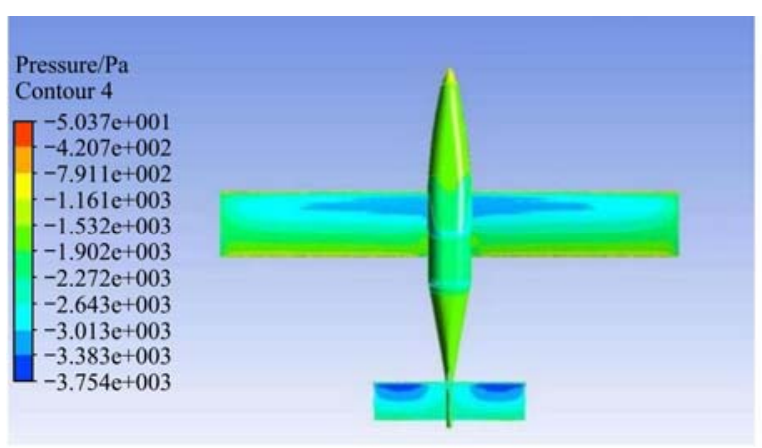

c. Below the wing

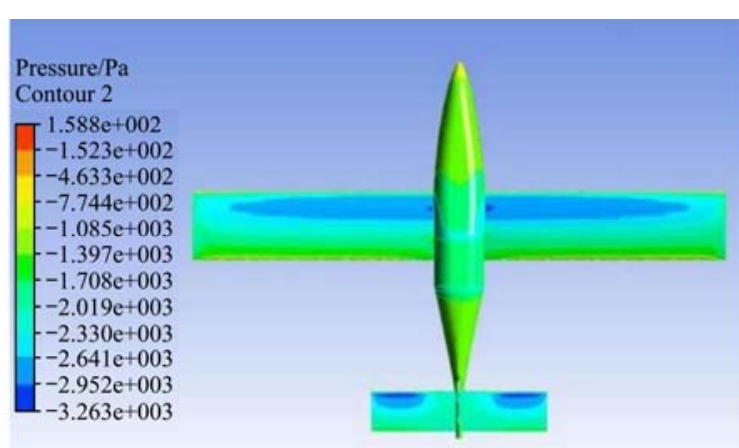

d. The rear wing

Figure 11 Aircraft pressure diagram with angle of attack of $0^{\circ}$ and pneumatic impeller at different positions

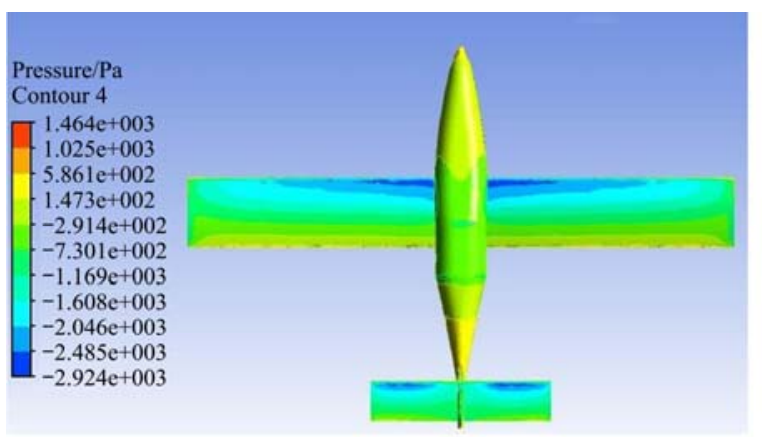

a. No impeller

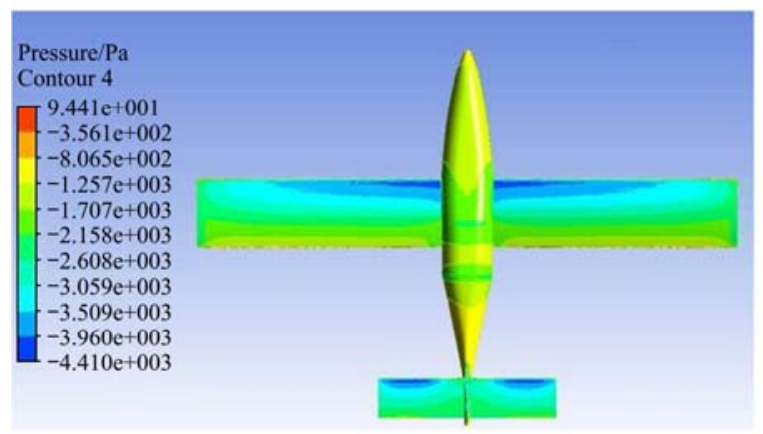

c. Below the wing

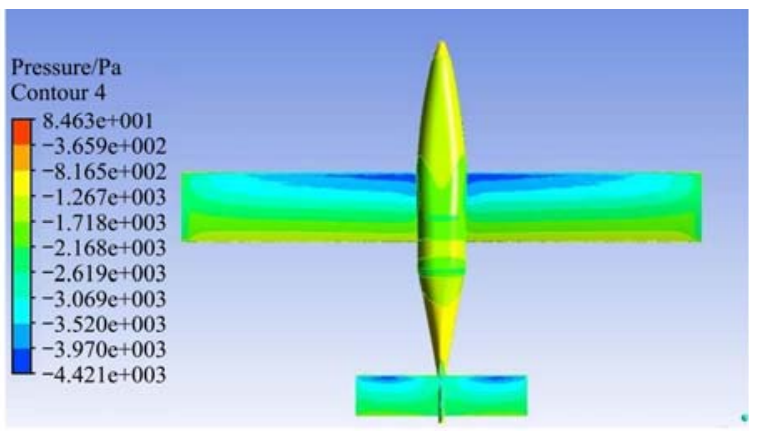

b. The front wing

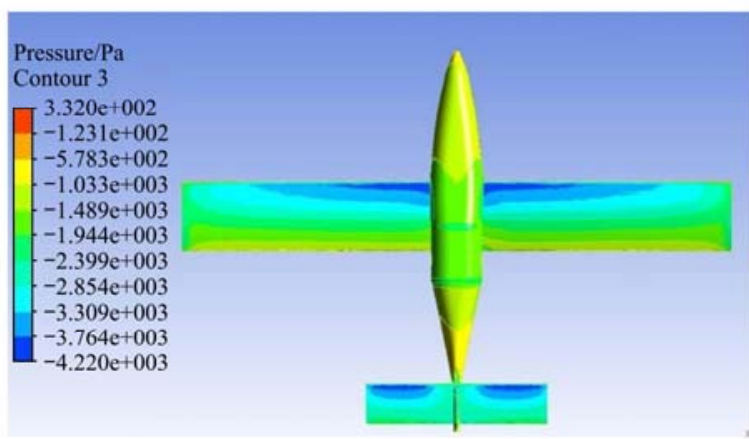

d. The rear wing

Figure 12 Aircraft pressure diagram with angle of attack of $6^{\circ}$ and pneumatic impeller at different positions

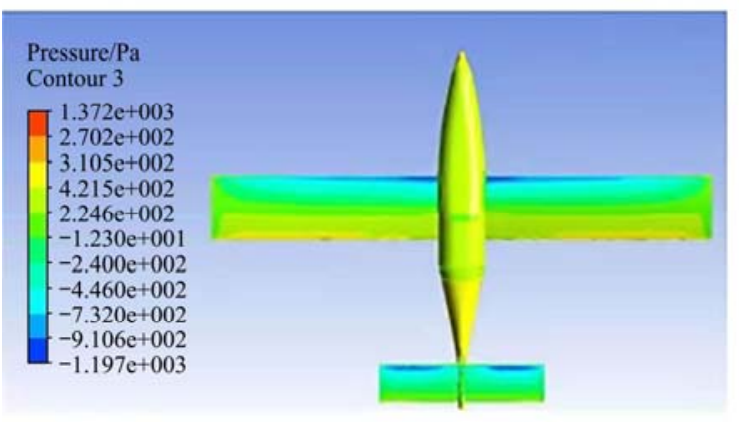

a. No impeller

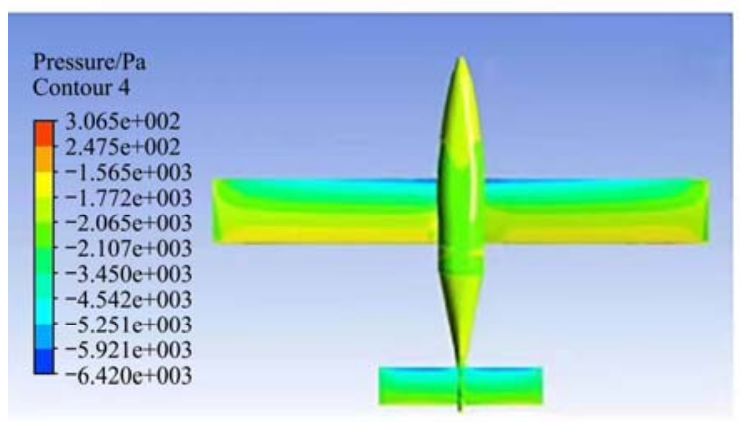

c. Below the wing

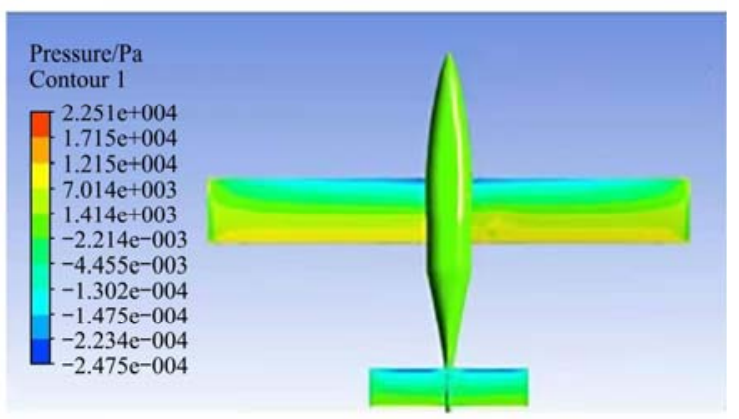

b. The front wing

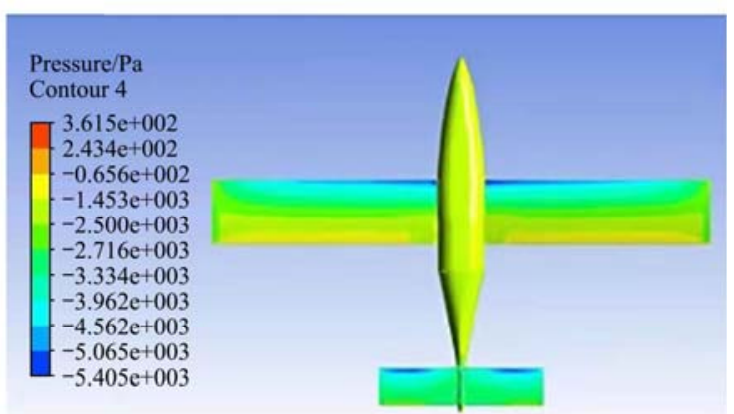

d. The rear wing

Figure 13 Aircraft pressure diagram with angle of attack of $12^{\circ}$ and pneumatic impeller at different positions 


\subsection{Influence of the installation position of the pneumatic impeller on itself}

Different installation positions of the pneumatic impeller will also have an impact on itself, and the output power of the pneumatic impeller at different installation positions is shown in Figure 14. It can be seen from the figure, no matter where the pneumatic impeller is located, the output torque continues to decrease with the increase of the angle of attack. As shown in Figure 15, the large pressure area on the windward side of the pneumatic impeller at an angle of attack of $0^{\circ}$ is much larger than that at an angle of attack of $12^{\circ}$. Therefore, the pneumatic impeller should be opened at a small angle of attack to collect more wind energy to improve the energy conversion efficiency of the pneumatic impeller. But when installed in pneumatic impeller under the wing output torque minimum and different angles of attack, the biggest change in the front wing with maximum output torque and different angles of attack change when the youngest, pneumatic impeller best location for the front wing, both to reduce the influence of aerodynamic characteristics of aircraft pneumatic impeller, in the case of large angle of attack is more conducive to the aircraft flight, at the same time, it reduces the influence of agricultural aircraft on the pneumatic impeller, so that the pneumatic impeller always keeps the appropriate output torque.

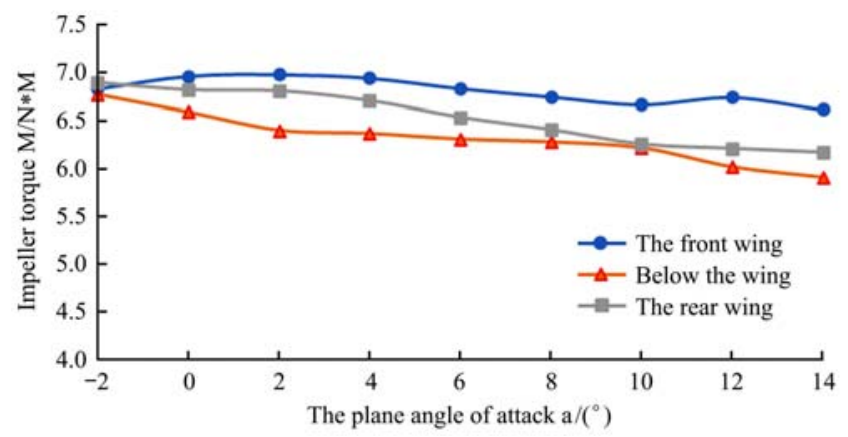

Figure 14 Output torque diagram of pneumatic impeller at different positions and angles of attack
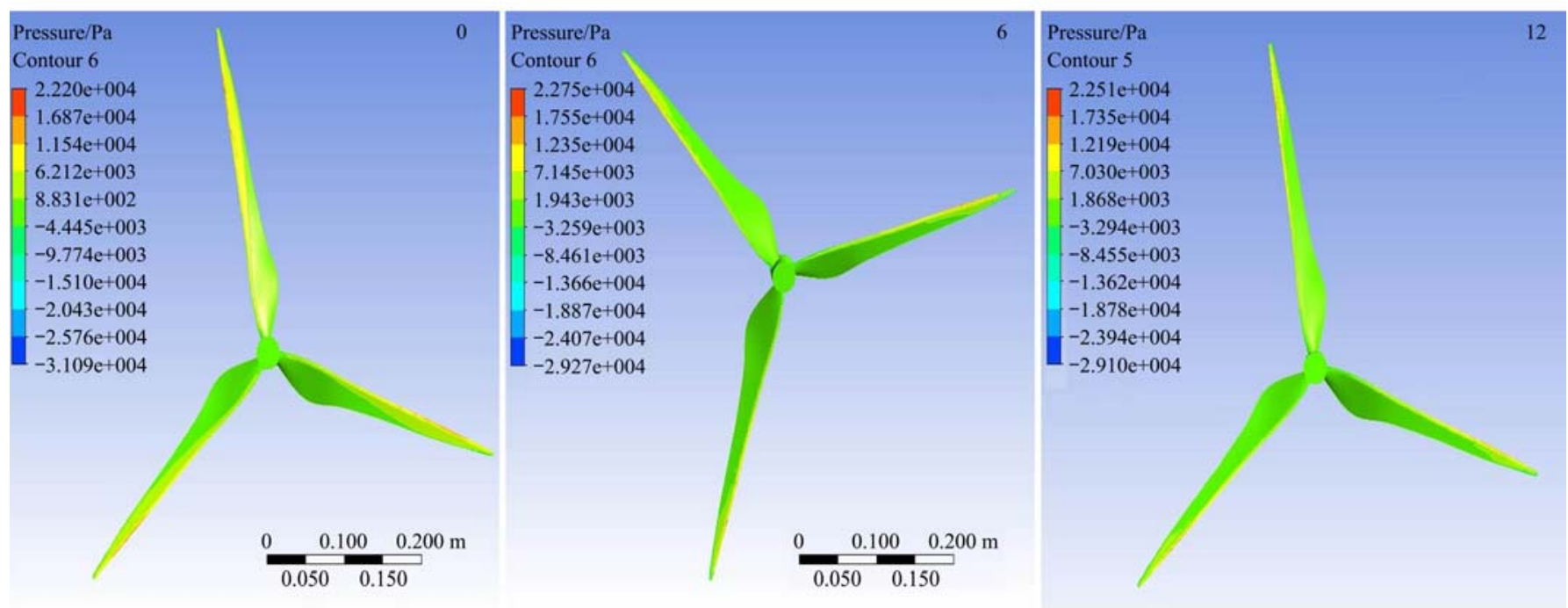

Figure 15 Pressure diagram of windward surface of pneumatic impeller at different angles of attack

4.4 Analysis of the interference of agricultural aircraft on the impeller under different wind speeds and angles of attack

Based on the previous analysis, the optimal installation position of the air impeller is obtained. The pneumatic impeller is installed in front of the wing, and the inlet wind speed in front is set at $40 \mathrm{~m} / \mathrm{s}, 50 \mathrm{~m} / \mathrm{s}$ and $60 \mathrm{~m} / \mathrm{s}$, and analysis the output power of the pneumatic impeller.

Calculation state: the atmospheric state is the standard atmospheric state near the ground, the angle of attack of the aircraft is calculated as $-2^{\circ}$ to $14^{\circ}$, the incoming wind speed is $40 \mathrm{~m} / \mathrm{s}$, $50 \mathrm{~m} / \mathrm{s}$ and $60 \mathrm{~m} / \mathrm{s}$ respectively, the installation position of the pneumatic impeller is in front of the wing, and the speed of the pneumatic impeller is set to $7000 \mathrm{r} / \mathrm{min}$. A total of 27 examples are carried out. After the calculation, with the rotating center of the impeller as the center, the output torque of the impeller is calculated to get the output torque diagram under different wind speeds and different angles of attack.

As can be seen from Figure 16, the output torque of the pneumatic impeller increases with the increase of the wind speed and decreases with the increase of the angle of attack. When the speed is constant, in order to obtain high output power, the flight angle of attack of the aircraft should be small and the speed should not be less than $50 \mathrm{~m} / \mathrm{s}$, which is in line with the general situation of agricultural aircraft. Agricultural aircraft will encounter a high angle of attack only in the process of take-off. During operation, it is generally in a flat flight state with an angle of attack of $0^{\circ}$ and a flying speed of $50-61 \mathrm{~m} / \mathrm{s}^{[16-20]}$.

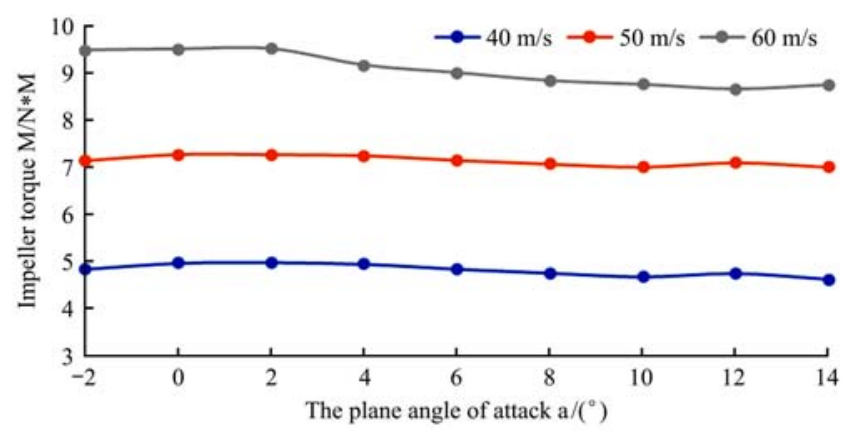

Figure 16 Output torque diagram of pneumatic impeller at different wind speed and aircraft angle of attack

Figure 17 shows the rotating flow charts of a single wind impeller and a front wing wind impeller with a flying speed of $50 \mathrm{~m} / \mathrm{s}$ at 0 angle of attack. According to the flow chart, the presence of the aircraft changes the direction of the air flow into the pneumatic impeller to a certain extent, and the tangential velocity of the air flow appears. Eddy currents appear in the flow out of the pneumatic impeller, leading to the change of the force on the pneumatic impeller and the reduction of the output torque. 


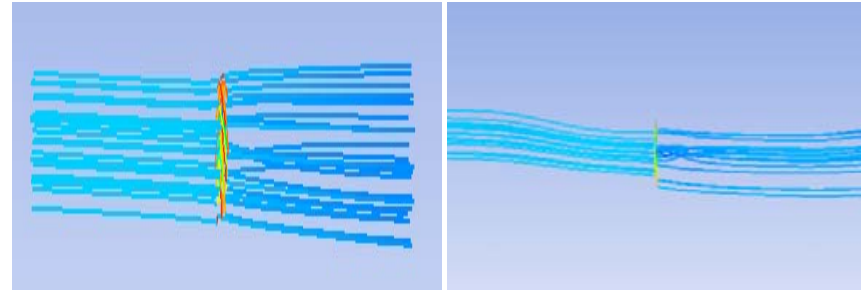

Figure 17 Rotating streamlines of individual pneumatic impeller (left) and wing front pneumatic impeller rotation flow chart (right)

\section{Conclusions}

Because the wind energy generated by agricultural aircraft during flight is very considerable, in order to make full use of this part of wind energy and reduce the energy consumption of agricultural aircraft, a pneumatic energy storage system for driving aircraft operation is designed. This paper analyzes and discusses the influence of the installation position of the pneumatic device on the aircraft, obtains the best installation position, reduces the interaction between the aircraft and the pneumatic device to the greatest extent, which has great significance to improve the energy utilization efficiency of the aircraft.

(1) By comparing the influence of the pneumatic impeller mounted under the wing, in front of the wing and behind the wing respectively on the lift coefficient and drag coefficient of agricultural aircraft, it is found that the pneumatic impeller installed in front of the wing can reduce the drag coefficient and increase the lift coefficient at the same time.

(2) The influences of the existence of the pneumatic impeller at 0,6 and 12 angles of attack and the different installation positions on the aircraft pressure distribution were analyzed. The results show that there is no pneumatic impeller under 0 angle of attack greatly reduced the minimum pressure wing surface area, more conducive to flight, with the increase of angle of attack, the installation position of different pressure distribution has no significant influence on the aircraft.

(3) The output torques of the pneumatic impeller at different installation positions at 0,6 and 12 angle of attack were compared respectively. It was found that the output torques decreased continuously with the increase of the angle of attack, but the output torques of the pneumatic impeller in front of the wing reached the maximum and changed slightly with the angle of attack.

(4) Comparing the output torques of the pneumatic impeller installed in front of the wing at different wind speeds of $40 \mathrm{~m} / \mathrm{s}$, $50 \mathrm{~m} / \mathrm{s}$ and $60 \mathrm{~m} / \mathrm{s}$ and at different angles of attack from $-2^{\circ}$ to $14^{\circ}$, it is concluded that the output torques increase with the increase of wind speed and decrease with the increase of angle of attack.

\section{Acknowledgments}

This study was supported by Top Talents Program for One Case One Discussion of Shandong Province and Program of Shandong Provincial Collaborative Innovation Center of Dry-farming Intelligent Agricultural Equipment and Academy of Ecological Unmanned Farm (2019ZBXC200).

\section{[References]}

[1] Wang X F. Progress of agricultural aviation technology in China. Modern Agriculture, 2013(08): 1-4.

[2] Pei S Y, Wang S Z, Zhang H H, Zhu H. Methods for monitoring and controlling multi-rotor micro-UAVs position and orientation based on LabVIEW. Int J Precis Agric Aviat, 2018; 1(1): 51-58. doi: 10.33440/ j.ijpaa.20180101.0009.

[3] Zhang B Z. Italy launched a new wind energy electric Tilt-rotor Prover. China Electric (Technical Edition), 2013, \{4\}(03): 3.

[4] Pathak R, Barzin R, Bora G C. Data-driven precision agricultural applications using field sensors and Unmanned Aerial Vehicle (UAVs). Int J Precis Agric Aviat, 2018; 1(1): 19-23. doi: 10.33440/j.ijpaa. 20180101.0004.

[5] The Netherlands Xsens company developed wind power uav. Inner Mongolia electric power technology, 2017,35(01):50.

[6] Tang W C. Analysis on the application of pneumatic pump technology in aircraft system. Machine Tool and Hydraulics, 2015, 43(16): 111-112+ 131.

[7] Li L, Yu J Z, Xie Y Q. Design of Small High Pressure Centrifugal Fan for Helicopter. Journal of Beijing University of Aeronautics and Astronsutics, 2003, (03): 244-247. doi: 10.13700/j.bh.1001-5965.2003. 03.015 .

[8] Gao H, Zhu P S, Gao Z H. Advanced Flight Dynamics. Beijing: National Defense Industry Press, 2004.

[9] Bao F. Geometrical Design and Aerodynamic Simulation of Wind Turbine Blade. Dalian University of Technology, 2009.

[10] Xu H R, Yang H, Liu C, Hong Z D. Aerodynamic Performance Prediction Accuracy of MEXICO Wind Turbine based on Different Turbulence Models. Proceedings of the CSEE, 2013, 33(35): 95-101+16. doi: 10.13334/j.0258-8013.pcsee.2013.35.014.

[11] Yu F H, Feng S A, Yao W X, Wang D K, Xing S M, Xu T Y. BAS-ELM based UAV hyperspectral remote sensing inversion modeling of rice canopy nitrogen content. Int J Precis Agric Aviat, 2020; 3(3): 59-64. doi: $+10.33440 /$ j.ijpaa.20200303.105.

[12] Wang B. Numerical Simulation and Optimization of Advanced Rotor Aerodynamic Characteristics Based on CFD Method. Nanjing University of Aeronautics and Astronautics, 2012.

[13] Ji F Z, Zhang M J, Wang R, Wang Y, Du F R. Blade design and aerodynamic performance numerical simulation on ram air turbine. Journal of Beijing University of Aeronautics and Astronautics, 2018, 44(07): 1387-1394.

[14] Zhang K K. Aerodynamic Performance and Vane Fluid-structure Coupling Analysis of 5MW Wind Turbine. Lanzhou University of Technology, 2019.

[15] Xue H B, Zhang X J, Chen H S, Xu Y J, Li W, Tan C Q. . Analysis of energy release process of micro-compressed air energy storage systems. Journal of Engineering Thermophysics, , 2014, 35(10): 1923-1929.

[16] Technical Regulations for the Control of White Moth by Aircraft Application (Lu Quality supervision Standard issued (2012) No. 45).

[17] Xue X Y, Lan Y B. Agricultural aviation technology status and development trend analysis in the United States. Transactions of the Chinese Society for Agricultural Machinery, 2013, 44(05): 194-201.

[18] Hong J B,Lan Y B, Yue X J, Cen Z Z, Wang L H, Peng W, Lu Y. Adaptive target spray system based on machine vision for plant protection UAV. Int J Precis Agric Aviat, 2020; 3(3): 65-71. doi: +10.33440/ j.ijpaa.20200303.92.

[19] Fritz B K, Hoffmann W C. Establishing reference nozzles for classification of aerial application spray technologies. Int J Precis Agric Aviat, 2018; 1(1): 10-14. doi: 10.33440/j.ijpaa.20180101.0003.

[20] Yao W X, Lan Y B, Wang J, Wen S, Wang G B, Chen S D. Droplet drift characteristics of aerial spraying of AS350B3e helicopter Transactions of the Chinese Society of Agricultural Engineering, 2017, 33(22): 75-83. 